\title{
IDENTIFICAÇÃO DE FATORES CRÍTICOS PARA ANÁLISE DA AGILIDADE EM ECOSSISTEMAS DE INOVAÇÃO
}

Silvia Ronsom (ronsom@gmail.com) - Universidade de São Paulo, Departamento de Engenharia de Produção - EESC-USP - São Carlos-SP, Brasil.

Daniel Capaldo Amaral ( Engenharia de Produção - EESC-USP - São Carlos-SP, Brasil.

\section{RESUMO}

Agilidade organizacional é uma habilidade de adaptação das organizações, reconhecida em ambientes de alta complexidade e volatilidade. Ela tem sido citada como relevante para explicar a dinâmica interna e a competição nos ecossistemas de inovação. No entanto, não há estudos descrevendo como o conceito de agilidade se relaciona com os ecossistemas e quais fatores determinam a agilidade em sua complexa rede de atores. Apresenta-se revisão bibliográfica e análise de conteúdo que identifica fatores que podem ser utilizados para avaliar a agilidade em ecossistemas. Como resultado, 20 fatores foram classificados em três níveis de análise: programas e portfólio, organização e ecossistema, indicando possíveis caminhos para o desenvolvimento da agilidade nesses ambientes.

Palavras chave: Agilidade, Ecossistemas de Inovação, Fatores críticos.

\section{INTRODUÇÃO}

O conceito de "ecossistemas de inovação" é cada vez mais importante para compreensão da nova realidade fundamentada na inovação e conectividade. Essa dinâmica tem exigido capacidade de adaptação das organizações (SULL, 2009; OCDE, 2015).

A agilidade pode ser entendida como uma habilidade de adaptação e autores sugerem que ela poderia explicar diferenças de desempenho entre ecossistemas que competem entre si (RUBENS et al., 2011; IANSITI; EUCHNER, 2018). Tal habilidade seria, portanto, fundamental para explicar a dinâmica de competição entre estes ecossistemas.

Apesar desta importância, não foram encontrados estudos que mediram ou observaram o construto agilidade em ecossistemas. Em uma busca para esta pesquisa, realizada entre abril e junho de 2020, não foram encontrados levantamentos em campo descrevendo fatores ou estratégias de agilidade em ecossistemas de inovação. 
Uma das barreiras é a ausência de um ferramental teórico, isso é, a identificação de um conjunto de fatores que caracterizem a agilidade e permitam a realização de estudos de caso para que se possa compreender este fenômeno no campo.

O objetivo desta pesquisa foi identificar o conjunto de fatores relacionados com a agilidade em ecossistemas de inovação em níveis de análise que emergem da literatura.

\section{REVISÃO TEÓRICA}

\subsection{A evolução da agilidade: da manufatura aos ecossistemas}

O termo "agilidade" tem sido discutido em todo o mundo como uma maneira de ganhar competitividade e melhorar as capacidades de inovação dos ambientes a que se destinam (SULL, 2009). As referências primárias desta pesquisa ${ }^{1}$ focam em áreas de conhecimento específicas, como por exemplo: agilidade em organizações de manufatura, agilidade no processo de desenvolvimento de produto e no gerenciamento de projetos.

Na manufatura, o termo "manufatura ágil" foi caracterizado como uma capacidade de alterar a configuração de um sistema em resposta a mudanças imprevistas e inesperadas condições de mercado (GOLDMAN; NAGEL; PREISS, 1995; GUNASEKARAN, 1999; SHARIFI; ZHANG, 2001).

No processo de desenvolvimento de produto, a agilidade vem sendo adotada para a melhoria dos métodos gerenciais (COOPER; EDGETT, 2009; CONFORTO; AMARAL, 2016). No gerenciamento de projetos é bastante difundida, com trabalhos como: Beck et al. (2001); Cohn (2005); Conforto et al. (2016).

Há uma tendência de adoção das metodologias ágeis e do conceito de agilidade em grande escala (DINGSØYR; MOE, 2014; PAASIVAARA et al., 2013). Rigby et al. (2016), afirmaram que os métodos ágeis estariam em expansão e maduros para serem utilizados em grande escala em empresas de diversas áreas.

No setor público, a agilidade estratégica é considerada fator fundamental de adaptação frente aos desafios e oportunidades do cenário socioeconômico (OCDE, 2015). Dentre estes estudos de agilidade no âmbito mais amplo, encontram-se artigos que analisam os ecossistemas de inovação.

\footnotetext{
${ }^{1}$ Confira Cooper (2009); Goldman, Nagel e Preiss (1995); Gunasekaran (1999); Sharifi e Zhang (2001).
} 


\subsection{O papel da agilidade nos ecossistemas de inovação}

Um ecossistema de inovação, segundo Carayannis e Campbell (2009, p. 202) é um conjunto de "pessoas, cultura e tecnologia interagem para catalisar a criatividade, desencadear invenções e acelerar a inovação através das disciplinas científicas e tecnológicas, dos setores público e privado, tanto em estratégias top-down, orientadas por políticas, quanto por intervenções bottom-up, baseadas em empreendedorismo". Emprega-se esta definição por destacar a colaboração público-privada, uma realidade que está muito relacionada a colaboração e inovação tecnológica, foco deste trabalho.

Dentre inúmeras configurações, há ecossistemas que envolvem atividades de Pesquisa e Desenvolvimento (P\&D), conforme sugeridos por Nambisan e Baron (2013) e aqueles que estudam um conjunto de entidades, como centros de pesquisa, que colaboram para prever e desenvolver inovações, como apontado por Zahra e Nambisan (2012).

Mais recentemente, vem se descobrindo a importância do aspecto agilidade nesses ambientes. Por exemplo, observa-se que a agilidade é um meio pelo qual as "espécies" fundamentais (key stones) podem se utilizar para adequar sua atuação e manter o ecossistema em funcionamento. Isso permite a evolução dos outros atores e seu comprometimento com o ecossistema (IANSITI; EUCHNER, 2018).

Exemplos como Tesla Motors e Bosch indicam este fenômeno. A Tesla adota práticas ágeis de testes constantes de ideias entre parceiros para resolver rapidamente seus problemas (STRINGHAM; MILLER; CLARK, 2015). A Bosch possui um programa para implementar métodos rápidos de validação de modelos de negócios em grande escala, o que contribui para o sucesso de seu portfólio de inovação (OSTERWALDER et al., 2020).

Os casos citados mostram como atores de um ecossistema podem influenciar o entorno de suas plataformas tecnológicas, criando competitividade e contribuindo para a evolução do seu ecossistema, o que pode conferir uma "agilidade" maior ao ecossistema como um todo. Mas, quais fatores precisam ser observados para que ela possa ser descrita ou mensurada?

\section{METODOLOGIA}

A pesquisa iniciou com a elaboração de um projeto de pesquisa e o estudo de fontes primárias sobre Ecossistemas, como, por exemplo, o de Iansiti e Euchner (2018), Gomes et al. (2018), Walrave et al. (2018) e Carayannis e Campbell (2009). Na área de Gestão Ágil, a definição de agilidade de Conforto et al. (2016) foi o ponto de partida, complementada pelos trabalhos de Beck et al. (2001), Boehm e Turner (2004), Amaral et al. (2011). 
O procedimento adotado para a RBS (Revisão Bibliográfica Sistemática) foi baseado em Conforto et al. (2011). Buscou-se identificar os principais elementos conceituais relacionados à agilidade e, respectivamente, organizações de pesquisa, ecossistemas de inovação e gestão de inovação, nas bases científicas (Web of Science e Scopus). Foi realizada uma seleção de artigos envolvendo tais termos em títulos e resumo. Foram realizadas leituras e análises de conteúdo para 51 artigos, conforme apresentado na Tabela 1.

Tabela 1- Quantidade de artigos lidos para a RBS

\begin{tabular}{|l|c|c|c|}
\hline Pesquisa & WoS & Scopus & Total \\
\hline Agilidade "E” organizações de pesquisa & 9 & 14 & 23 \\
\hline Agilidade "E” Ecossistemas de inovação & 8 & 13 & 21 \\
\hline Agilidade "E” Gestão de inovação & 3 & 4 & 7 \\
\hline Total & 20 & 31 & 51 \\
\hline
\end{tabular}

Fonte: elaborada pelos autores.

Empregou-se a análise dedutiva e técnica de codificação aberta para a análise de conteúdo. A codificação aberta significa que as notas e os títulos são escritos no texto durante a leitura, o material é lido quantas vezes forem necessárias, todos os aspectos do conteúdo são descritos e as categorias são livremente geradas (ELO; KYNGÄS, 2007).

\section{RESULTADOS E DISCUSSÃO}

\section{1 - Fatores de agilidade e os níveis de análise}

A análise do conjunto de artigos selecionados permitiu identificar padrões para agilidade, classificados em 3 subníveis:

- Subnível 1. Programas e portfólios. Referências identificadas para o gerenciamento de programas e portfólios de projetos em ambientes de ecossistemas.

- Subnível 2. Organizações. Estudos relacionados a organizações de pesquisa, centros de pesquisa e eventuais startups de inovação que interagem em ecossistemas.

- Subnível 3. Ecossistema. Envolvem ambientes amplos e complexos de inovação, sendo admitidos, por exemplo, grupos de agentes auto-organizados e centros de pesquisas de empresas com atuação descentralizada e ou global.

\section{2 - Fatores de agilidade no subnível de Programas e Portfólios}

Douthwaite e Hoffecker (2017) apresentam atividades de adaptação às mudanças para programas de pesquisa no setor agrícola: 1) Consenso para desafios abrangentes; 2) Revisão 
regular de visões, questões de pesquisa, planos e progressos realizados; 3) Atuação e decisão autônoma de grupos e plataformas para resolução de problemas em diferentes escalas.

Sweetman e Conboy (2018) apresentam proposições para gerenciamento de portfólios de projetos ágeis baseadas em Sistemas Adaptativos Complexos, com foco nas seguintes propriedades: auto-organização; propósito comum; autonomia; adaptabilidade; variedade de requisitos; troca de recursos.

No ambiente indústria-academia, Sandberg, Pareto e Arts (2011) apresentaram 10 princípios de ação: focar em garantir resultados; assegurar engajamento da gestão; abraçar negociações de pesquisa; organizar encontros; comunicar progresso e resultado constantemente; atender às necessidades e aos objetivos; ser ágil, admitir entregas frequentes; financiar pequenos projetos; inovação proveniente das necessidades; aprendizado.

Vidmar (2019) e Vidmar et al. (2020) relatam adoção de abordagens de gerenciamento de projetos, envolvendo parceiros de pesquisa, usuários principais e outras partes interessadas (financiadores, reguladores, etc.) em diferentes conjuntos de projetos.

Conforto e Amaral (2016) apresentam dados empíricos sobre o uso e o efeito de um framework (híbrido) para gestão de projetos em empresas de base tecnológica. Os resultados estão sintetizados no Quadro 1.

Quadro 1 - Fatores de agilidade para Programas e portfólios

\begin{tabular}{|l|l|}
\hline Fatores de agilidade & Referências \\
\hline $\begin{array}{l}\text { Alinhamento de uma visão ou mensagem essencial } \\
\text { de agilidade }\end{array}$ & $\begin{array}{l}\text { Sandberg, Pareto e Arts (2011); Douthwaite e } \\
\text { Hoffecker (2017) }\end{array}$ \\
\hline $\begin{array}{l}\text { Aplicação de métodos e técnicas (Exemplos: gates; } \\
\text { pesquisa participativa; histórias de usuários) }\end{array}$ & $\begin{array}{l}\text { Cooper (2014); Douthwaite e Hoffecker (2017); } \\
\text { Sweetman e Conboy (2018) }\end{array}$ \\
\hline $\begin{array}{l}\text { Frameworks ou plataformas colaborativas de de } \\
\text { softwares }\end{array}$ & $\begin{array}{l}\text { Cooper (2009); Gonzalez (2014); Conforto e Amaral } \\
\text { (2016); Cooper (2014). }\end{array}$ \\
\hline
\end{tabular}

Fonte: elaborado pelos autores com base no conjunto de artigos identificados na literatura.

\section{3 - Fatores de agilidade no subnível de organizações}

Shin et al. (2015), afirmam que em organizações, a agilidade estratégica influencia positivamente no alinhamento interno ou "consenso estratégico". Chikhale e Mansouri (2015) apontam a importância de construir consenso e confiança, pela identificação de grupos de stakeholders e desenvolvimento de planos de ação.

Liu e Yang (2020) defendem que as organizações que buscam agilidade devem operar menos por regras e mais baseadas em princípios, favorecendo o comportamento empreendedor. 
Teoh e Cai (2015) comentam que o processo de antecipação e resposta como componentes de agilidade estão associados com capacidades de antecipação, transformação de conhecimento, harmonização para resolver problemas e acomodar mudanças, interação com clientes e adaptabilidade.

Propostas de frameworks e uso de plataformas colaborativas foram identificadas, por exemplo em Weiblen e Chesbrough (2015). Eles apresentarm uma tipologia de modelos de engajamento corporativo com startups e seus principais objetivos: Programa de inicialização (Outside-In e plataforma); Incubação Corporativa; Negócio corporativo. O resultado está sintetizado no Quadro 2.

Quadro 2 - Fatores de agilidade no subnivel Organizações

\begin{tabular}{|c|c|}
\hline Fatores & Referências \\
\hline $\begin{array}{l}\text { Alinhamento de uma visão (ou mensagem } \\
\text { essencial) de agilidade }\end{array}$ & $\begin{array}{l}\text { Cross (2015); Shin et al. (2015); Chikhale e Mansouri } \\
\text { (2015); Liu e Yang (2020) }\end{array}$ \\
\hline $\begin{array}{l}\text { Aplicação de métodos e técnicas (Exemplos: } \\
\text { conquista de partes interessadas relevantes; } \\
\text { equilíbrio entre inovação e gestão de risco) }\end{array}$ & $\begin{array}{l}\text { Teoh e Cai (2015); Vanharanta et al., (2018); Liu e Yang } \\
\text { (2020) }\end{array}$ \\
\hline Aprendizado contínuo ou by design & Pérez-Bustamante (1999); Teoh e Cai, 2015 \\
\hline Comportamento empreendedor & Liu e Yang (2020) \\
\hline $\begin{array}{l}\text { Desenvolvimento de mentalidade e capacidades } \\
\text { ágeis }\end{array}$ & $\begin{array}{l}\text { Pérez-Bustamante (1999); Mihardjo } \text { et al. (2019); } \\
\text { Kuivalainen, Kunttu e Kohtamaki (2020) }\end{array}$ \\
\hline Desenvolvimento de colaboração e aprendizado & Pérez-Bustamante (1999); Shin et al. (2015); Vidmar (2019) \\
\hline Desenvolvimento de lideranças & $\begin{array}{l}\text { Meyer; Marion (2010); Krstic, Skorup e Lapcevic (2018); } \\
\text { Liu e Yang (2020) }\end{array}$ \\
\hline $\begin{array}{l}\text { Frameworks ou plataformas colaborativas de } \\
\text { softwares }\end{array}$ & $\begin{array}{l}\text { Calof; Smith (2010); Meyer e Marion (2010); Cross (2015); } \\
\text { Shin et al. (2015); Weiblen e Chesbrough (2015); Chikhale; } \\
\text { Mansouri (2015); Adegbile, Sarpong e Meissner (2017); } \\
\text { Houngbe, Barthe-Delanoe e Negny (2019); Liu; Yang } \\
(2020)\end{array}$ \\
\hline Implementação gradual ou em estágios & Teoh e Cai (2015); Cross (2015); Fritzsch et al. (2019); \\
\hline Troca de recursos nas redes & Meyer e Marion (2010); Liu e Yang (2020) \\
\hline
\end{tabular}

Fonte: elaborado pelos autores com base no conjunto de artigos identificados na literatura.

\section{4 - Fatores de agilidade no subnível do ecossistema}

Esta seção apresenta fatores que poderiam inspirar ações e decisões gerenciais em ecossistemas, onde estariam maiores desafios para agilidade.

Vidmar (2019) e Vidmar et al. (2020) apresentaram uma proposta de uso de uma mensagem central para inovação e colaboração intersetorial no desenvolvimento de produtos, serviços e suas infraestruturas de suporte para a Indústria Espacial na Escócia.

Klerkx, Arts e Leeuwis (2010) discutem a participação de brokers de inovação especializados para auxiliar na formulação e reformulação da visão e, adaptação contínua de uma rede de atores, impactando na agilidade. 
A coordenação de plataformas foi observada por Argyropoulou, Soderquist e Ioannou (2019), que defenderam a importância de uma entidade ágil que gerencia uma variedade de campos científicos e coordena variados mecanismos de financiamento. Chikhale e Mansouri (2015) apresentam ideias semelhantes, porém focadas no caso da Apple.

Weinberg (2019) descreveu uma perspectiva histórica sobre a mudança do papel do apoio governamental em ecossistemas empresariais em Israel. O processo inclui a introdução de agências de apoio público/privado destinadas a ser mais flexíveis e ágeis para atender às necessidades de empresas, clusters e ecossistemas em constante mudança.

Katzy e Crowston (2008) concluíram que a agilidade é o resultado de atividades empreendedoras apoiadas por rotinas organizacionais, padrões de comportamento recorrente que são compartilhados por parceiros da rede em geral. Kuivalainen, Kunttu e Kohtamaki (2020) consideram práticas ágeis para lidar com um paradoxo de aprendizagem em unidades de P\&D em cadeia global de empresas.

Mergel (2016), em pesquisa para gerenciamento da inovação ágil a nível nacional, se baseou em três princípios: a) inovação aberta para o desenvolvimento de softwares; b) necessidade de uma abordagem de liderança ágil; c) abordagens alternativas de contratação e iterações que permitam identificar pequenas falhas para rápida correção.

$\mathrm{O}$ autor apresentou uma proposta de abordagem ágil incluindo: camada de base (políticas); camada de gerenciamento guarda-chuva (baseada em metodologias ágeis), de gerenciamento de processos e liderança, destacando os agile BPAs (Blanket Purchase Agreements) e RFP (Requests for Proposal). Os resultados estão sintetizados no Quadro 3.

Quadro 3 - Fatores de agilidade no subnível Ecossistema

\begin{tabular}{|c|c|}
\hline Fatores operacionais & Referências \\
\hline $\begin{array}{l}\text { Alinhamento de uma visão (ou mensagem } \\
\text { essencial) de agilidade }\end{array}$ & $\begin{array}{l}\text { Klerkx, Arts e Leeuwis (2010); Chikhale e Mansouri } \\
\text { (2015); Mergel (2016); Vidmar (2019); }\end{array}$ \\
\hline Coordenação de plataformas & $\begin{array}{l}\text { Klerkx, Arts e Leeuwis (2010); Argyropoulou, } \\
\text { Soderquist e Ioannou (2019); Weinberg (2019) }\end{array}$ \\
\hline Comportamento empreendedor & Katzy e Crowston (2008) \\
\hline Desenvolvimento de colaboração e aprendizado & $\begin{array}{l}\text { Klerkx, Arts e Leeuwis (2010); Katzy e Crowston } \\
\text { (2008); Shin et al. (2015); Kuivalainen, Kunttu e } \\
\text { Kohtamaki (2020); Vidmar et al. (2020) }\end{array}$ \\
\hline $\begin{array}{l}\text { Frameworks ou plataformas colaborativas de } \\
\text { softwares }\end{array}$ & Mergel (2016) \\
\hline $\begin{array}{l}\text { Aplicação de métodos e técnicas (Exemplos: } \\
\text { BPAs; análise participativa de caminho crítico; } \\
\text { monitoramento de processo reflexivo) }\end{array}$ & Mergel (2016); Klerkx, Arts e Leeuwis (2010) \\
\hline Troca de recursos nas redes & Liu e Yang (2020) \\
\hline
\end{tabular}


Fonte: elaborado pelos autores com base no conjunto de artigos identificados na literatura.

\section{CONCLUSÃO}

$\mathrm{O}$ artigo contribui com a identificação dos fatores que podem determinar a agilidade de um ecossistema de inovação segundo a literatura da área. Os fatores foram classificados em três subníveis de análise: Programas e Portfólios, Organizações e Ecossistemas. Ao todo são 20 fatores que podem ser avaliados por pesquisadores interessados em ampliar a compreensão do tema.

Os próximos passos desta pesquisa envolvem a realização de uma análise exploratória destes fatores em um ecossistema de inovação para que no futuro seja possível identificar um quadro teórico que descreva a agilidade em ecossistemas de inovação.

\section{REFERÊNCIAS}

ADEGBILE A.; SARPONG D.; MEISSNER D. Strategic foresight for innovation management: A review and research agenda. International Journal of Innovation and Technology Management. 2017.

AMARAL, D.; CONFORTO, E.; BENASSI, J-L.; ARAÚJO, C. Gerenciamento ágil de projetos: aplicação em produtos inovadores. São Paulo: Saraiva. 2011.

ANDRIOPOULOS C., LEWIS M. Exploitation-Exploration Tensions and Organizational Ambidexterity: Managing Paradoxes of Innovation. Organization Science. Vol. 20, No. 4. 2008.

ARGYROPOULOU M., SODERQUIST K.E., IOANNOU G. Getting out of the European Paradox trap: Making European research agile and challenge driven. European Management Journal. v. 37, p. 1-5, 2019.

BECK K. et al. Manifesto for agile software development. 2001. Disponível em <https://agilemanifesto.org/iso/ptbr/manifesto.html>. Acessado em 10/02/2020.

BOEHM, B., TURNER, R., 2004. Balancing agility and discipline: A guide for the perplexed addison-Wesley, Boston. 2004.

CARAYANNIS, E.G., CAMPBELL, D.F.J., 'mode 3' and 'quadruple helix': toward a 21st century fractal innovation ecosystem. Int. J. Technol. Manag. v. 46, n.3-4, p. 201-234. 2009.

CHIKHALE M.; MANSOURI M. An Agile and Collaborative Framework for Effective Governance to Enhance Management in Large-Scale Enterprise Business Systems: The Case of Apple Inc. Global Journal of Flexible Systems Management. Vol. 16, p. 283-293. 2015.

COHN, M., Agile Estimating and Planning. Prentice Hall PTR, New York. 2005.

CONFORTO E.; AMARAL D. C. Agile project management and stage-gate model-A hybrid framework for technology-based companies. J. Eng. Technol. Management. v. 40, p. 1-14, 2016.

CONFORTO E.; AMARAL D. C; Silva S. L. Roteiro para revisão bibliográfica sistemática: aplicação no desenvolvimento de produtos e gerenciamento de projetos. 8o Congresso Brasileiro de Inovação e Gestão do Desenvolvimento de Produto (CBGDP). Anais.. Porto Alegre - RS. 2011.

CONFORTO, E.; AMARAL D.C.; SILVA, S.; FELIPPO, A.; KAMIKAWACHI, D. The agility construct on Project management theory. International Journal of Project Management. v. 34, p. 660-674, 2016.

COOPER, R. G. Managing technology development projects. Research Technology Management, v. 49, n. 6, nov./dez., 2006.

COOPER, R.; EDGETT, S. Product Innovation and Technology Strategy. United States: Product and Development Institute Inc. 2009.

COOPER, R.G. What's next? After stage-gate. Research Technology Management. v. 57, p. 20-31, 2014. 
CREVELING, C. et al.,. Design for six sigma: in technology \& product development. New Jersey: Prentice Hall PH, 2003.

CROSS S.E. A model to guide organizational adaptation. 2013 International Conference on Engineering Technology and Innovation ICE 2013 and IEEE International Technology Management Conference. 2015.

DINGSØYR, T., MOE, N. Towards principles of large-scale agile development. In: Dingsøyr, T., Moe, N., Tonelli, R., Counsell, S., Gencel, C., Petersen, K. (Eds.), Agile Methods. Large-Scale Development, Refactoring, Testing, and Estimation. In: Lecture Notes in Business Information Processing, vol. 199. Springer International Publishing, pp. 1-8.2014.

DOUTHWAITE B.; HOFFECKER E. Towards a complexity-aware theory of change for participatory research programs working within agricultural innovation systems. Agricultural Systems. v. 155, p. 88-102, 2017.

DUPONT, L. Agile innovation: Creating value in uncertain environments. Journal of Innovation Economics \& Management. v. 28, p. 1-5, 2019.

ELO S.; KYNGAS H. The qualitative content analysis process. JAN Research Methodology 2007.

FRITZSCH, J., BOGNER, J., WAGNER, S., ZIMMERMANN, A.. Microservices Migration in Industry: Intentions, Strategies, and Challenges. Proceedings - 2019 IEEE International Conference on Software Maintenance and Evolution. 2019.

GOLDMAN, S.L., NAGEL, R.N., PREISS, K. Agile Competitors and Virtual Organizations: Strategies for Enriching the Customer. Van Nostrand Reinhold, New York. 1995.

GOMES L. A. V.; FACIN A.L.; SALERNO M. S.; IKENAMI R. K. Unpacking the innovation ecosystem construct: Evolution, gaps and trends. Technological Forecasting \& Social Change. v. 136, p. 30-48, 2018.

GONZALEZ, W. Applying Agile Project Management to Predevelopment. International Journal of Innovation and Technology Management. Vol 11 (04). 2014.

GUNASEKARAN, A., Agile manufacturing: a framework for research and development. International Journal of Production Economics. v. 62, p. 87-105. 1999

HIGHSMITH, J. Agile Project Management: creating innovative products. Addison-Wesley: Boston, 2004.

HOUNGBE, M; BARTHE-DELANOE, AM; NEGNY, S A systemic approach for agile biorefineries. 29TH EUROPEAN SYMPOSIUM ON COMPUTER AIDED PROCESS ENGINEERING, PT B. 2019.

IANSITI, M.; EUCHNER, J. Competing in Ecosystems. Research-Technology Management, v.61, n. 2, p. 10$16,2018$.

KATZY, BR; CROWSTON, K. Competency rallying for technical innovation - The case of the Virtuelle Fabrik. Technovation. v. 28, p. 679-692, 2008.

KLERKX, L; ARTS, N; LEEUWIS, C. Adaptive management in agricultural innovation systems: The interactions between innovation networks and their environment. Agricultural Systems. v. 103, p. 390-400, 2010.

KRSTIC, M; SKORUP, A; LAPCEVIC, G. Trends In Agile Innovation Management. International Review. v. 3, p. 58-70, 2018.

KUIVAlAinEN, J; KUNTTU, I; KOHTAMAKI, M. Agile Product Development Practices for Coping with a Learning Paradox in R\&D Offshore Units. Technology Innovation Management Review, v. 10, n. 3, 2020.

LEWIS, M. W. Exploring paradox: Toward a more comprehensive guide. The Academy of Management Review, v. 25, n. 4, p. 760-776, 2000.

LIU H-M.; YANG H-F. Network resource meets organizational agility: Creating an idiosyncratic competitive advantage for SMEs. Management Decision. 2020.

MERGEL I. Agile innovation management in government: A research agenda. Government Information Quarterly. Vol. 33, 3, p. 516-523, 2016.

MIHARDJO L.; SASMOKO S.; ALAMSYAH F.; ELIDJEN D. Boosting the Firm Transformation in Industry 5.0: Experience-Agility Innovation Model International Journal of Recent Technology and Engineering (IJRTE). Vol. 8, Issue-2S9, 2019. 
NAMBISAN, S.; BARON, R.A. Entrepreneurship in innovation ecosystems: Entrepreneurs' self-regulatory processes and their implications for new venture success. Enterprise Theory Pract. v. 37, n. 5, p. 1071-1097. 2013.

OCDE - ORGANIZAÇÃO PARA A COOPERAÇÃO E DESENVOLVIMENTO ECONÔMICO. Achieving Public Sector Agility at Times of Fiscal Consolidation. OECD Public Governance Reviews, OECD Publishing. 2015.

OSTERWALDER A. et al. The invincible company. John Wiley \& Sons, Inc., Hoboken, New Jersey. 2020.

PAASIVAARA, M., LASSENIUS, C., HEIKKILA, V., DIKERT, K., ENGBLOM, C., Integrating global sites into the lean and agile transformation at ericsson. In: Global Software Engineering (ICGSE), 2013 IEEE 8th International Conference on, 134-14, 2013.

PÉREZ-BUSTAMANTE, G. Knowledge management in agile innovative organisations. Journal of Knowledge Management. v. 3, p. 6-17, 1999.

RIGBY D.; SUTHERLAND J.; TAKEUCHI H. Embracing Agile. Harvard Business Review. 2016.

RONSOM S; AMARAL D. Avaliando o potencial de aplicação do conceito de agilidade na gestão de tecnologia em ICTs: o caso EMBRAPII. 12o Congresso Brasileiro de Inovação e Gestão do Desenvolvimento de Produto (CBGDP). Anais.. Brasília - DF. 2019.

RUBENS, N., STILL, K., HUHTAMÄKI, J., RUSSELL, M.G., A network analysis of investment firms as resource routers in Chinese innovation ecosystem. J. Softw. v. 6, n. 9, p. 1737-1745, 2011.

SANDBERG, A., PARETO, L., ARTS, T. Agile collaborative research: Action principles for industry-academia collaboration. IEEE Software. 2011.

SHARIFI, H., ZHANG, Z. Agile manufacturing in practice. Application of a methodology. Int. Journal of Operations and Production Manag. v.21, p. 772-794, 2001

SHIN, H; LEE, JN; KIM, D; RHIM, H. Strategic agility of Korean small and medium enterprises and its influence on operational and firm performance. International Journal of Production Economics. 2015

STRINGHAM E. P.; MILLER J. K.; CLARK J.R. Overcoming Barriers to Entry in an Established Industry: Tesla Motors. California Management Review, Vol. 57, n. 4, 2015.

SULL, D., 2009. Competing through organizational agility. McKinsey Q. December, 2009: Disponível em <http://www.mckinsey.com/insights/managing_in_incerainty/competing_through_organizational_agility> Acessado em: 20/02/2020.

SWEETMAN R.; CONBOY K. Portfolios of Agile Projects: A Complex Adaptive Systems' Agent Perspective. Project Management Journal. v. 49, n. 6, p. 18-38, 2018.

TAVANI S.N.; SHARIFI H.; ISMAIL H.S. A study of contingency relationships between supplier involvement. International Journal of Operations and Production Management. 2014.

TEOH, S.Y.; CAI, S. The process of strategic, agile, innovation development: a healthcare systems implementation case study. Journal of Global Information Management, Vol. 23 No. 3, pp. 1-22. 2015.

VANHARANTA, H; KANTOLA, J; MARKOPOULOS, E; SALO, M; EINOLANDER, J; HANHISALO, T. The Degree of Agility In a technology company's strategy, management, and leadership. Management and Production Engineering Review. 2018.

VIDMAR M.; ROSIELLO A.; VERMEULEN N.; WILLIAMS R.; DINES J. New Space and Agile Innovation: Understanding transition to open innovation by examining innovation networks and moments. Acta Astronautica. 2020.

VIDMAR, M. Agile Space Living Lab - The Emergence of a New High-Tech Innovation Paradigm. Space Policy. v. 9, n. 101324, 2019.

WALRAVE B.; TALMAR M.; PODOYNITSYNA K. S.; ROMME A. G. L.; VERBONG GEERT P.J. A multilevel perspective on innovation ecosystems for path-breaking Innovation. Technological Forecasting \& Social Change. Vol. 136, p. 103-113. 2018.

WEIBLEN T.; CHESBROUGH H.W. Engaging with startups to enhance corporate innovation. California Management Review, v. 57, n. 2, 2015. 
WEINBERG C. Entrepreneurial Ecosystems in Israel: The Changing Role of Government Support: A Historical Perspective. Portland International Conference on Management of Engineering and Technology (PICMET), Portland, OR, USA, pp. 1-3, 2019.

WILSON K.; DOZ Y. Agile Innovation: A footprint balancing distance and immersion. California Management Review. v. 53, n .2, p. 6-26, 2011.

ZAHRA, S.A; NAMBISAN, S. Entrepreneurship and strategic thinking in business ecosystems. Business Horizons. v. 55, n. 3, p. 219-229, 2012. 